Sharif University of Technology
Scientia Iranica
SCIENTIA

\title{
Numerical simulation of non-Newtonian nanofluids flow in passive injection micromixers using mixture model
}

\author{
S. Baheri Islami ${ }^{a, *}$, A. Alizad ${ }^{a}$ and R. Gharraei ${ }^{b}$ \\ a. Faculty of Mechanical Engineering, University of Tabriz, Tabriz, Iran. \\ b. Department of Mechanical Engineering, Azarbaijan Shahid Madani University, Tabriz, Iran. \\ Received 11 May 2015; received in revised form 14 December 2015; accepted 2 May 2016
}

\section{KEYWORDS}

Non-Newtonian fluid; Nanofluid;

Injection micromixer;

Mixture model;

Passive micromixer.

\begin{abstract}
In this study, mixing of laminar non-Newtonian nanofluids in an injection micromixer was numerically studied. The important and new feature of the study is using of non-Newtonian base fluid in nanofluid. The Titanium dioxide/0.5\%wt Carboxymethyl Cellulose aqueous solution was used as nanofluid. Mixture model was used for simulation of nanofluid flow inside the micromixer. The governing equations were solved by finite volume method using a FORTRAN code. Also, in this paper, a new modified successive over-relaxation method has been introduced to considerably decrease the computation time. The results indicated that the number of injection flows and using of baffles had significant effect on mixing index. It should be noted that the distance between baffles is an important parameter in mixing.
\end{abstract}

(C) 2017 Sharif University of Technology. All rights reserved.

\section{Introduction}

Miniaturization of fluid analysis has been developed in the interdisciplinary research field of microfluidics during the past two decades. Some of the microfluidic applications are micro arrays, DNA sequencing, sample preparation and analysis, cell separation, and detection and environmental monitoring. The use of microfluidics in these applications attracts interest from both industry and academia because of its advantages, i.e. small amounts of sample, less time consumption, lower cost, and high throughput. Rapid mixing is essential in many microfluidic systems used in biochemistry analysis, drug production, and synthesis of nucleic acids. Biological processes often involve reactions that require rapid and complete mixing of reactants for initiation. Mixing is also necessary in

* Corresponding author. Tel.: +984133392458; E-mail addresses: baheri@tabrizu.ac.ir (S. Baheri Islami); aamech87@gmail.com (A. Alizad); gharraei@azaruniv.ac.ir (R. Gharraei)
Lab-On-a-Chip (LOC) systems for complex chemical reactions [1].

Because of the low Reynolds numbers and, consequently, the laminar flow in microchannels, the dominant mixing mechanism is molecular diffusion. Therefore, rapid mixing is impossible and effective mixing needs large channel lengths and long mixing times. Thus, it is necessary to find some methods to enhance the mixing phenomena.

Among two major kinds of micromixers (active and passive), passive ones attract more interest because of their simplicity and low cost. Some very simple passive methods have been presented in this study.

There are many numerical and experimental studies about mixing of fluids using micromixers. Engler et al. [2] investigated mixing of liquids in static micromixers by computational and experimental methods. They concluded that increasing vorticity inside static T-shaped micromixers with rectangular crosssections occurred, even at low Reynolds numbers, and these effects could be used to improve the mixing index. Galletti et al. [3] numerically studied the effect 
of entrance conditions on the engulfment pattern in a T-shaped micromixer. They observed that if the flows at the micromixer confluence were not fully developed, engulfment occurred at larger Reynolds numbers with a different flow pattern. In these cases, mixing indices were lower than those in the case where the entrance flows were fully developed. Alam and Kim [4] investigated mixing in a curved micromixer with rectangular grooves; the results showed that grooved micromixers produced better mixing quality than smooth micromixers at Reynolds numbers greater than 10. Afzal and Kim [5] evaluated mixing efficiency in a passive split and recombination micromixer with convergent-divergent walls by numerical methods. The proposed micromixer gave significantly improved mixing performance compared to a similar geometry based on the concept of unbalanced split and collisions for a wide range of Reynolds numbers. Bhagat et al. [6] studied mixing in a passive planar micromixer with obstructions for mixing at low Reynolds numbers. The design incorporated diamond-shaped obstructions within the micromixer to break-up and recombine the flow. Ansari et al. [7] introduced a novel passive micromixer based on the concept of unbalanced splits and cross-collisions of fluid streams. In this paper, the three-dimensional Navier-Stokes equations were solved numerically to analyze the mixing and flow behavior of the micromixer, which was composed of two sub-channels of unequal widths. Soleymani et al. [8] studied liquid mixing in T-type micromixers, numerically. Simulation results indicated that the development of vortices was crucial to achieve good mixing performance.

Wang et al. [9] investigated a rapid magnetic particle-driven micromixer, numerically. In this paper, effects of magnetic actuation force, switching frequency, and lateral dimension of mixer were investigated. Fu et al. [10] numerically analyzed rapid micromixer via ferrofluids; they observed that the external magnetic field caused the ferrofluid to expand significantly and uniformly toward miscible water, associated with a great number of extremely fine fingering structures, on the interface in the upstream and downstream regions of the micromixer. Along with the dominant diffusion effects occurring around the circumferential regions of the fine finger structures, the mixing efficiency improves significantly.

Bhagat and Papautsky [11] studied particle dispersion enhancement in a passive planar micromixer using rectangular obstacles, numerically and experimentally. The performance of the micromixer was benchmarked against a conventional Y-mixer and the modified Tesla mixer, which had been shown to work well with fluids.

Baheri et al. [12] investigated hydrodynamics and heat transfer of $\mathrm{CuO} / \mathrm{CMC}$ solution non-Newtonian nanofluids in micromixers with baffle. They observed that the main factor of enhancing heat transfer or mixing was the recirculation zones that were created behind the baffles. Hadigol et al. [13] studied electroosmotic micromixing of non-Newtonian fluids numerically. The results confirmed that the sheardependent viscosity had a significant effect on the degree of mixing efficiency. It was shown that as the fluid behavior index of power-law fluid decreased, more homogeneous solution could be achieved at the microchannel outlet.

There is not any numerical study on the mixing of non-Newtonian nanofluids using micromixers with twophase models with non-Newtonian base fluids. Also, the presented mixing methods based on the division and distributions of nanofluids are very simple and low-cost passive methods. Therefore, in the present study, mixing and pressure drop of $\mathrm{TiO}_{2} / \mathrm{CMC}$ solution nanofluid in a passive injection micromixer with two injection flows from bottom and top walls have been investigated. Nanofluid flow has been modeled with a mixture model using a FORTRAN code.

\section{Geometry}

The geometry of the proposed micromixer can be seen in Figure 1. The two-dimensional micromixer is composed of two parallel plates with two injection flows from bottom and top walls and two zero-thickness baffles. The height and the length of micromixer are

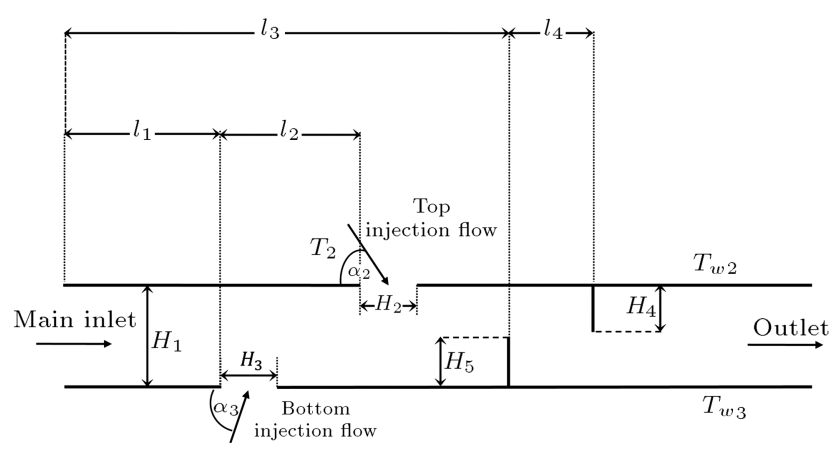

Figure 1. Geometry of micromixer. 
$H_{1}=100 \mu \mathrm{m}$ and $20 H_{1}$, respectively. The distance of bottom injection flow from channel inlet and the distance between injections have been shown with $l_{1}$ and $l_{2}$, respectively. The distance of the lower baffle from channel inlet, the distance between baffles, and heights of top and bottom baffles have been shown with $l_{3}, l_{4}, H_{4}$, and $H_{5}$, respectively. The angles of top and bottom injection flows with respect to the channel wall have been named $\alpha_{2}$ and $\alpha_{3}$, respectively. Also, the widths of top and bottom entrances have been shown with $\mathrm{H}_{2}$ and $\mathrm{H}_{3}$, respectively.

\section{Governing equations and boundary conditions}

\subsection{Governing equations}

As we know, the flow is a continuum flow for Knudsen numbers (ratio of mean free path to characteristic length) smaller than $10^{-3}$ and it is accurately modeled by the Navier-Stokes equations with classical no-slip boundary conditions. The fluid in this study is liquid and for liquid molecules, the mean free path is 0.1$1 \mathrm{~nm}$ [14]. Since the smallest size in this study is $100 \mu \mathrm{m}$, the flow is in continuum regime.

In the present study, it is assumed that the suspended nanoparticles and the base liquid are in local thermal equilibrium and the Brownian diffusion and thermophoresis are the only mechanisms by which the nanoparticles develop a significant relative velocity with respect to the base liquid. Thus, any particlerelated effect is not accounted for [15]. Mixture model has been used in order to simulate the nanofluid flow. The governing equations are continuity, momentum, energy, and volume fraction, which can be written as follows:

$$
\begin{aligned}
& \nabla \cdot\left(\rho_{n f} \overrightarrow{V_{n f}}\right)=0 \\
& \nabla \cdot\left(\rho_{n f}\left(V_{n f}\right)_{j} \overrightarrow{V_{n f}}\right)=-\vec{\nabla} P+\nabla \cdot\left(\eta_{n f} \vec{\nabla}\left(V_{n f}\right)_{j}\right), \\
& \nabla \cdot\left(\rho_{n f} \overrightarrow{V_{n f}} C_{p_{n f}} T_{n f}\right)=\nabla \cdot\left(k_{n f} \vec{\nabla} T_{n f}\right), \\
& \nabla \cdot\left(\rho_{p} \phi \overrightarrow{V_{n f}}\right)=\nabla \cdot\left(\rho_{n f} D_{b} \vec{\nabla}\left(\frac{\rho_{p}}{\rho_{n f}} \phi\right)\right) \\
& +\nabla \cdot\left(\rho_{n f} D_{t} \vec{\nabla} T\right)
\end{aligned}
$$

where $\overrightarrow{V_{n f}}$ is the mixture velocity vector; $\left(V_{n f}\right)_{j}$ is the $j$-component of mixture velocity; $\phi$ is the volume fraction of nanoparticles; $\rho_{n f}, \rho_{p}, \eta_{n f}, C_{p_{n f}}$, and $k_{n f}$ are mixture density, particle density, effective apparent viscosity, effective specific heat, and effective thermal conductivity, respectively. The nanoparticle diffusion mass flux is sum of the Brownian and thermophoretic diffusion terms in the hypothesis of dilute mixture (i.e., low volume fraction ). $D_{b}$ and $D_{t}$ are the Brownian and thermophoretic diffusion coefficients, respectively.

For the non-Newtonian Ostwald-de Waele fluid model, the relationship between apparent viscosity and shear rate is as follows [16]:

$$
\eta_{n f}=m\left[\left|\sqrt{\frac{1}{2} \overline{\dot{\gamma}}: \overline{\bar{\gamma}}}\right|^{n-1}\right],
$$

where $\overline{\bar{\gamma}}=\nabla V+\nabla V^{T}$ is shear rate tensor, and $m$ and $n$ are two empirical constants dependent on the type of non-Newtonian nanofluid. For two-dimensional fluid motion in rectangular coordinate system, the term $\frac{1}{2} \overline{\bar{\gamma}}: \overline{\bar{\gamma}}$ is defined as follows:

$$
\frac{1}{2} \overline{\dot{\gamma}}: \overline{\dot{\gamma}}=2\left\{\left(\frac{\partial u}{\partial x}\right)^{2}+\left(\frac{\partial v}{\partial y}\right)^{2}\right\}+\left(\frac{\partial v}{\partial x}+\frac{\partial u}{\partial y}\right)^{2} .
$$

The Reynolds number of each inlet has been calculated based on the related entrance width, velocity, and base fluid properties:

$$
\operatorname{Re}_{H_{i}}=\frac{\rho_{f} V_{i}^{2-n_{f}} H_{i}^{n_{f}}}{8^{n_{f}-1} m_{f}\left(\frac{3 n_{f}+1}{4 n_{f}}\right)^{n_{f}}},
$$

where $V_{i}$ and $H_{i}$ are the mean velocity and entrance width of inlet $i$, respectively. The subscript $i$ takes the values of 1,2 , or 3 for main flow inlet, top injection flow, or bottom injection flow, respectively. The subscript $f$ in Eq. (7) stands for base fluid. In order to evaluate the mixing efficiency, the variance of the mass fraction of nanoparticle within the mixture in a cross section, normal to the flow direction, is defined as:

$$
\sigma=\sqrt{\sum_{i=1}^{n} \frac{\left(x_{i}-x_{m}\right)^{2}}{N}}
$$

where $\sigma$ is the variance over the data range, $N$ is the total number of sampling points inside the cross section, $x_{i}$ is the mass fraction at the sampling point $i$, and $x_{m}$ is the complete mixing mass fraction defined as:

$$
x_{m}=\frac{\sum_{i=1}^{n} \rho_{i} V_{i} H_{i} x_{i}}{\sum_{i=1}^{n} \rho_{i} V_{i} H_{i}},
$$

where subscript $i$ depicts number of each inlet as mentioned above.

The number of sampling points $N$ for each cross sectional plane is taken as 1531 to ensure high accuracy [17]. The sampling points are equidistant on the cross sectional line. The mixing efficiency can be calculated as follows:

$$
\mathrm{DOM}=1-\sqrt{\frac{\sigma^{2}}{\sigma_{\max }^{2}}},
$$


where DOM is the mixing degree and $\sigma_{\max }$ is the maximum variance over the data range. Non-dimensional pressure drop can be defined as:

$$
\Delta p^{*}=-\frac{\Delta p}{\left(\rho_{f}\right)_{0} u_{1}^{2}} .
$$

$\Delta p$ is the pressure difference between channel outlet and inlet and subscript 0 shows the values obtained in reference temperature of $293 \mathrm{~K}$.

Another parameter, which has been used in the present study, is Reynolds number ratio. It is the ratio of injection Reynolds number to the main-flow Reynolds number and is defined as:

$$
\chi=\frac{n \operatorname{Re}_{H i}}{\operatorname{Re}_{H 1}},
$$

in which $n$ is the number of injection flows.

The other parameter is mass flow rate ratio. It is the ratio of total injection mass flow rate (from top plus bottom injection flows) to the main flow one and is defined as:

$$
m r=\frac{\sum_{i=1}^{n} \dot{m}_{i}}{\dot{m}_{1}}
$$

In the above equation, $n$ shows the total number of injections.

Also, non-dimensional lengths and velocity are defined as follows:

$$
\begin{aligned}
& U=\frac{u}{u_{1}}, \\
& X=\frac{x}{H_{1}}, \quad Y=\frac{y}{H_{1}}, \quad l_{1}^{*}=\frac{l_{1}}{H_{1}}, \quad l_{2}^{*}=\frac{l_{2}}{H_{1}}, \\
& l_{3}^{*}=\frac{l_{3}}{H_{1}}, \quad H_{4}^{*}=\frac{H_{4}}{H_{1}}, \quad H_{5}^{*}=\frac{H_{5}}{H_{1}} .
\end{aligned}
$$

$l_{1}^{*}$ is 5 for all of the cases and $\frac{H_{2}}{H_{1}}=\frac{H_{3}}{H_{1}}=0.5$.

\subsection{Boundary conditions}

The uniform velocity, temperature, and volume fraction profile have been applied for all of the inlets. Also, temperature and volume fraction profiles are uniform. For microchannel outlet, gradients of all variables (except pressure) have been assumed equal to zero.

The constant temperature and no-slip boundary conditions have been applied to the walls.

All of the mixing processes are isothermal at $293 \mathrm{~K}$, except in the case of temperature effect investigation.

\section{Thermophysical properties of nanofluid}

In this study, $\mathrm{TiO}_{2}$-CMC nanofluid has been used.
The effective density and the heat capacitance of nanofluid can be calculated according to the following equations [18]:

$$
\begin{aligned}
& \rho_{n f}=(1-\phi) \rho_{f}+\phi \rho_{p} \\
& \left(\rho C_{p}\right)_{n f}=(1-\phi)\left(\rho C_{p}\right)_{f}+\phi\left(\rho C_{p}\right)_{p} .
\end{aligned}
$$

Hojjat et al. [19] presented experimental relation between the shear stress and shear strain for $\mathrm{TiO}_{2} / \mathrm{CMC}$ nanofluid at different solid volume fractions and temperatures. Using this data, a two-variable surface was fitted to the values of $m$ and $n$ for $0 \%$ to $4 \%$ solid volume fractions and the temperature range of 288 to $308 \mathrm{~K}$. The variations of $m$ and $n$ by temperature and volume fraction of nanoparticles have been given in Eqs. (18) and (19). Also, Figure 2 depicts variations of $m$ and $n$ by volume fraction of nanoparticles at $T=293 \mathrm{~K}$. As can be seen, the nanofluid has shear thinning behavior.

$$
\begin{aligned}
m= & 0.2055-0.04446 T_{s v}+0.01588 \phi_{s v}+0.002864 T_{s v}^{2} \\
& -0.01117 \phi_{s v} T_{s v}+0.02276 \phi_{s v}^{2}+0.003665 \phi_{s v} T_{s v}^{2} \\
& -0.004874 T_{s v} \phi_{s v}^{2}+0.03606 \phi_{s v}^{3}, \\
n= & 0.5181+0.01468 T_{s v}-0.0133 \phi_{s v}+0.0002679 T_{s v}^{2} \\
& -0.002317 \phi_{s v} T_{s v}-0.001234 \phi_{s v}^{2}-0.001436 \phi_{s v} T_{s v}^{2} \\
& +0.001594 T_{s v} \phi_{s v}^{2}-0.003819 \phi_{s v}^{3},
\end{aligned}
$$

where:

$$
\begin{aligned}
\phi_{s v} & =\frac{\phi-0.019}{0.01478}, \\
T_{s v} & =\frac{T-298.1}{8.452} .
\end{aligned}
$$

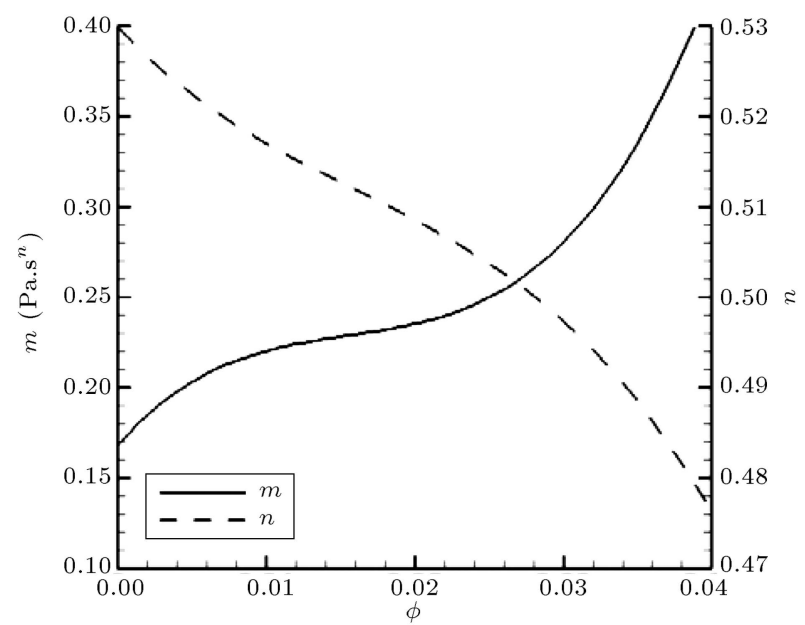

Figure 2. Variations of power law and consistency index versus volume fraction of nanoparticles, $T=293 \mathrm{~K}$. 


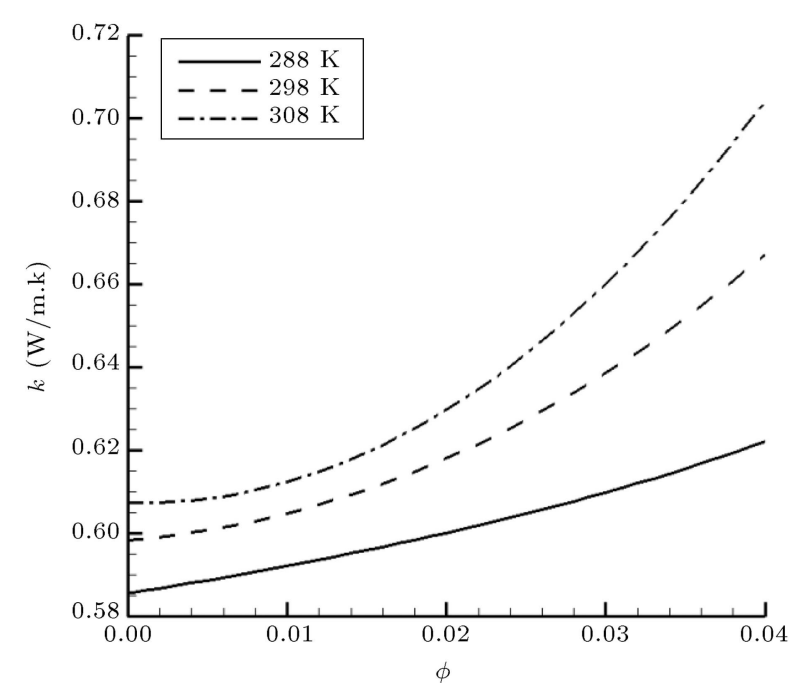

Figure 3. Variations of thermal conductivity of nanofluid versus volume fraction of nanoparticles for various temperatures.

The effective thermal conductivity of nanofluid has been adapted from the work of Hojjat et al. [20]. A two-variable surface has been fitted to the corresponding data. Variations of thermal conductivity of the nanofluid by temperature and volume fraction of nanoparticles have been shown in Eq. (22) and Figure 3.

$$
\begin{aligned}
k_{n f}= & 0.6091+0.009498 T_{s k}+0.014 \phi_{s k} \\
& -0.001973 T_{s k}^{2}+0.003881 \phi_{s k} T_{s k} \\
& +0.004541 \phi_{s k}^{2}-0.0004616 \phi_{s k} T_{s k}^{2} \\
& +0.003024 T_{s k} \phi_{s k}^{2}+0.0001553 \phi_{s k}^{3}
\end{aligned}
$$

where:

$$
\begin{aligned}
T_{s k} & =\frac{T-298.2}{8.528}, \\
\phi_{s k} & =\frac{\phi-0.01375}{0.01131} .
\end{aligned}
$$

All physical properties of CMC are assumed to be temperature-dependent. Polynomial curve fitting has been performed for CMC physical properties such as density and specific heat in the temperature interval of $293 \mathrm{~K}-303 \mathrm{~K}$.

$$
\begin{aligned}
& \rho_{f}=-0.35 T+1099.6025, \\
& C_{p f}=2.193 T_{s c}^{3}-2.633 T_{s c}^{2}-4.033 T_{s c}+4195,
\end{aligned}
$$

where:

$$
T_{s c}=\frac{T-301.1}{4.036}
$$

The Brownian diffusion coefficient $D_{b}$ is given by the Einstein-Stokes equation [21]:

$$
D_{b}=\frac{k_{b} T}{3 \pi \eta_{f} d_{p}},
$$

where $k_{b}=1.3806488 \times 10^{-23}$ is the Boltzmann constant, $\eta_{f}$ is apparent viscosity of base fluid, and $d_{p}$ is diameter of nanoparticles and is selected according to [19] and [20].

$D_{t}$ can be calculated using the McNab-Meisen relationship for the thermophoretic velocity of particles dispersed in liquids of [22], which is written as:

$$
D_{t}=0.26 \frac{k_{f}}{k_{f}+k_{p}} \frac{\eta_{f} \rho_{p}}{\rho_{f} \rho_{n f}} \frac{\phi}{T}
$$

\section{Numerical method}

The governing equations have been discretized using finite volume method [23]. Power-law scheme [24] for discretization of momentum and energy convective fluxes and QUICK scheme [23] for discretization of volume fraction convective fluxes have been used.

The pressure-velocity coupling algorithm was SIMPLE [23]. The set of discretized equations have been solved using line-by-line method [23]. In order to decrease the computational time, a novel SOR (Successive Over Relaxation) method has been used. In this method, over-relaxation factor has been set to 1 at the beginning. After each 30 sweeps, $\frac{0.004}{n}$ has been added to the over-relaxation factor in each line, in which $n$ is the number of lines. It has been continued until 200th sweeps. Then, $\frac{0.1374}{n}$ has been added to over-relaxation factor after each 30 sweeps until the 400th sweep. The computational time has decreased by more than $98 \%$ in comparison with the ordinary line-by-line method. Note that ordinary SOR method becomes stable only for coarse grids and, even in these cases, computation time is high. The values of increment of over-relaxation factor have been obtained by trial and error to reach an acceptable reduction in computational time.

The scaled residuals have been used as the convergence parameter. The scaled residuals smaller than $10^{-3}, 10^{-3}, 10^{-5}$, and $10^{-3}$ have been used for continuity, momentum, energy, and volume fraction, respectively, as the convergence criterion.

\section{Results}

\subsection{Grid independency test}

In order to check independency of the results from the number of cells, a grid independency test has been done by considering the calculated velocity profile in fully developed region for $\mathrm{CMC}$ solution flow in a 


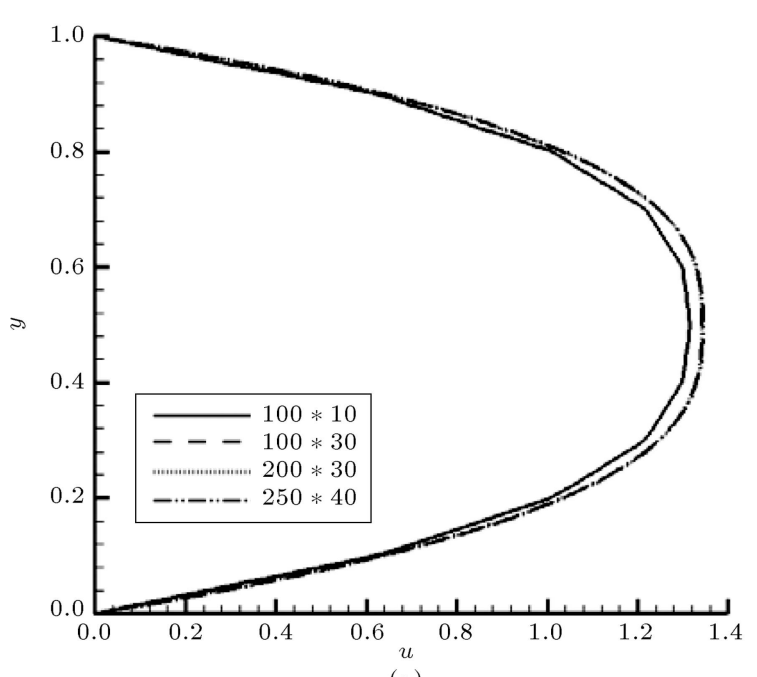

(a)

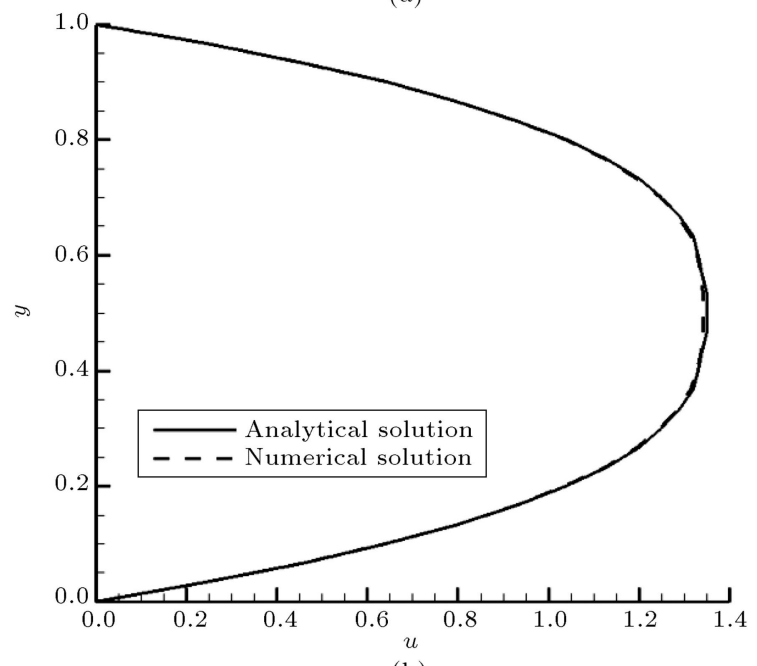

(b)

Figure 4. (a) Grid independency test. (b) Validation of fully developed non-dimensional velocity profile of CMC solution flow without nanoparticles between two infinite parallel plates, $\operatorname{Re}_{H_{1}}=1$.

microchannel without injection. The results are shown in Figure 4(a). It is clear that using finer grid does not have considerable effect on the results; thus, the $200 \times$ 30 grid is chosen for this case. The grid independency has been checked for other results in a similar manner.

\subsection{Code validation}

In order to validate the written code, fully developed velocity profile for the flow of CMC solution without nanoparticles $(\phi=0)$ and without injection has been compared with that of the analytical solution.

Figure 4(b) shows the non-dimensional fully developed velocity profile. It can be seen that the agreement between results is very good.

\subsection{The effect of number of injections and mass flow rate ratio}

In this section, the effect of mass flow rate ratio for single and double injections has been investigated. In

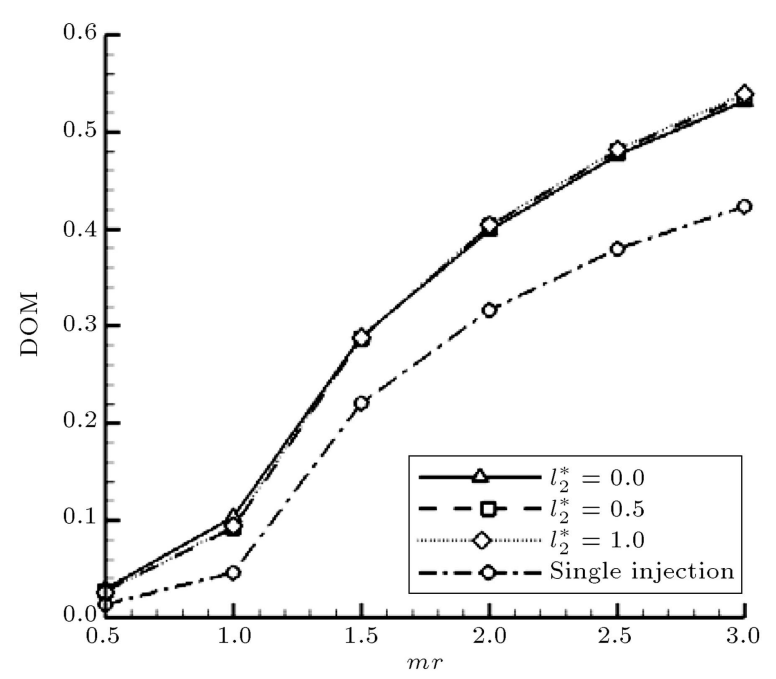

Figure 5. Variations of mixing degree versus mass flow rate ratio for single injection and double injection with various injector distances; $\dot{m}_{1}=10 \mu \mathrm{g} / \mathrm{S}, \phi_{1}=0$, $\phi_{2}=0.02$, and $\phi_{3}=0.02$.

the single injection, only bottom injection flow and in the double injection, both the top and bottom injection flows exist. In order to compare the single and double injection cases, the total mass flow rate of top and bottom injections is equal to the mass flow rate of single injection. Also, the mass flow rate of top and bottom injectors is equal to that of double injection. The Reynolds number of mainstream is $\operatorname{Re}_{H_{1}}=0.01$.

Figure 5 shows the effect of mass flow rate ratio and the distance between injection flows on degree of mixing.

It is clear from Figure 5 that mixing degree increases with increasing the mass flow rate ratio. Also, for the given mass flow rate ratio, the double injection has higher mixing degree than single injection because of the further contact surface of injection flows with mainstream and less mass fraction variance. Also, Figure 5 shows that distance between injectors in double injection does not have significant effect on mixing index. But, it is clear that mixing index decreases by further increment of the distance between injectors due to less diffusion path.

The abovementioned mixing behavior can be explained by Figure 6. In this figure, the nanoparticle volume fraction contours have been presented for two mass flow rate ratios. It is clear that as the distance between injections increases, diffusion path becomes larger; but, due to low Brownian diffusion coefficient, because of high apparent viscosity, this increment in diffusion path does not have significant effect on mixing index.

The effect of mass flow rate ratio on nondimensional pressure drop can be seen in Figure 7 . This figure shows increase in pressure drop with mass flow rate ratio for both single and double injections.

Also, pressure drop for double injection with 

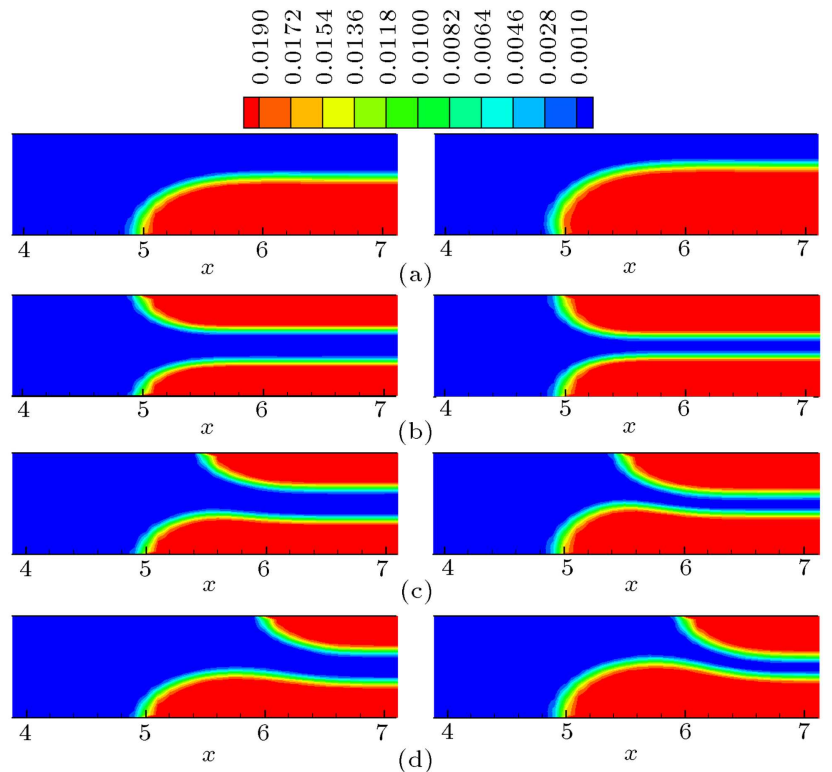

Figure 6. The contours of nanoparticle volume fraction: (a) Single injection, (b) double injection with $l_{2}^{*}=0.0$, (c) double injection with $l_{2}^{*}=0.50$, and d) double injection with $l_{2}^{*}=1, \dot{m}_{1}=10 \mu \mathrm{g} / \mathrm{S}, \phi_{1}=0, \phi_{2}=0.02$, and $\phi_{3}=0.02$ (left: $m r=1.5$, and right: $m r=3$ ).

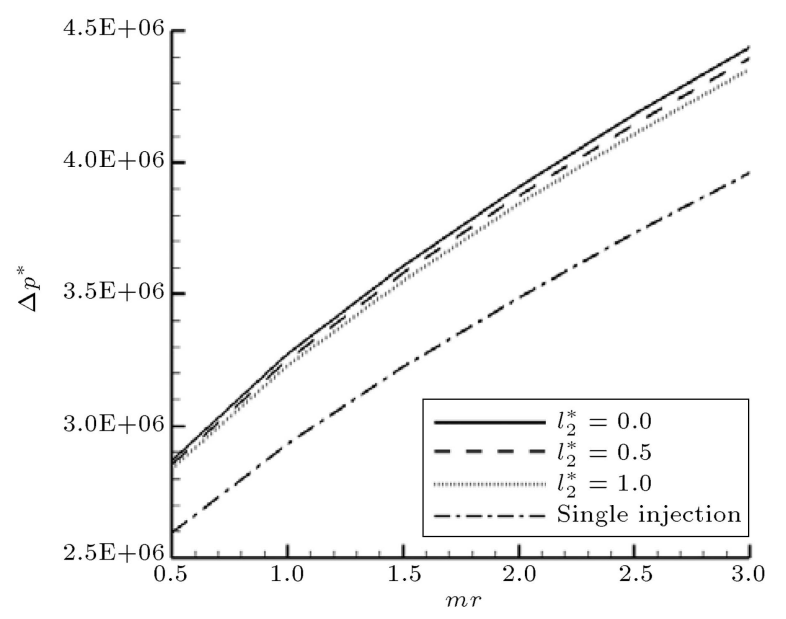

Figure 7. Variations of pressure drop versus mass flow rate ratio for single injection and double injection with various injector distances; $\dot{m}_{1}=10 \mu \mathrm{g} / \mathrm{S}, \phi_{1}=0$, $\phi_{2}=0.02$, and $\phi_{3}=0.02$.

$l_{2}^{*}=0.5$ or $l_{2}^{*}=1.0$ is less than that for $l_{2}^{*}=0$; because, in these cases, the resistance of injection flows against mainstream is lower than the case with $l_{2}^{*}=0$. Finally, the sensitivity of pressure drop to the number of injection flows and mass flow rate ratio is less than the one of mixing degree.

\subsection{The Effect of nanoparticle volume fraction}

In this section, the effect of volume fraction on degree of mixing and pressure drop has been studied. The results have been presented in Table 1 for a
Table 1. The effect of nanoparticles volume fraction on mixing degree and pressure drop, $\operatorname{Re}_{H_{1}}=0.01, \chi=4$, and $l_{2}^{*}=1.0$

\begin{tabular}{cccccc}
\hline $\begin{array}{c}\text { Test } \\
\text { number }\end{array}$ & $\boldsymbol{\phi}_{\mathbf{1}}$ & $\boldsymbol{\phi}_{\mathbf{3}}$ & $\boldsymbol{\phi}_{\mathbf{2}}$ & DOM & $\boldsymbol{\Delta} \boldsymbol{p}^{*}$ \\
\hline 1 & 0.0 & 0.05 & 0.0 & 0.0054 & 73262.2468 \\
2 & 0.0 & 0.04 & 0.01 & 0.1698 & 644674058 \\
3 & 0.0 & 0.03 & 0.02 & 0.3853 & 56467.8963 \\
4 & 0.0 & 0.02 & 0.03 & 0.3860 & 56333.9584 \\
5 & 0.0 & 0.01 & 0.04 & 01031 & 63874.9691 \\
6 & 0.0 & 0.0 & 0.05 & 0.0026 & 70961.0599 \\
7 & 0.03 & 0.0182 & 0.0182 & 0.3986 & 57222.6847 \\
\hline
\end{tabular}

different mainstream with top and bottom injection volume fractions at constant mainstream and injection Reynolds numbers. In all cases, mean mass fraction of nanoparticles $\left(x_{m}\right)$ has been maintained at a constant value, but mass flow rate ratio can vary case by case. It can be seen that as the nanoparticles are distributed by almost equal volumetric flows from all main inlets and injectors (Test numbers 3, 4, and 7 in Table 1), mixing degree and pressure drop become higher and lower, respectively, than those in other cases. As the variations of nanoparticles volume fraction become smaller within micromixer, the mass fraction of nanoparticles gets closer to the mean mixing degree; therefore, the DOM improves. The highest DOM is for test number 7 , in which volume fraction distribution is smoother than other cases. The apparent viscosity near walls becomes smaller due to less volume fraction in this region and, consequently, shear stress at the wall; therefore, pressure drop decreases. The effect of equal nanoparticles distribution is more considerable on the degree of mixing than on pressure drop.

Volume fraction contours for three different cases have been illustrated in Figure 8. It can be inferred that volume fraction distribution of nanoparticles is more uniform for the test number 7 than for other cases.

\subsection{The effect of temperature dependent properties}

All results of the previous section were presented in isothermal condition, but the physical properties, such as Brownian diffusion coefficient and viscosity of base fluid, have been assumed temperature-dependent in this study. Because of the high value of apparent viscosity, thermal diffusion coefficient is considerable.

The temperature dependency of thermophysical properties and thermal diffusion can affect the flow patterns and, subsequently, mixing process. In order to compare the results of this section with isothermal results, the mass flow rate ratio has been assumed equal to the isothermal mixing at $m r=3$ for all cases. The definition of $\chi$ cannot be used in this section 


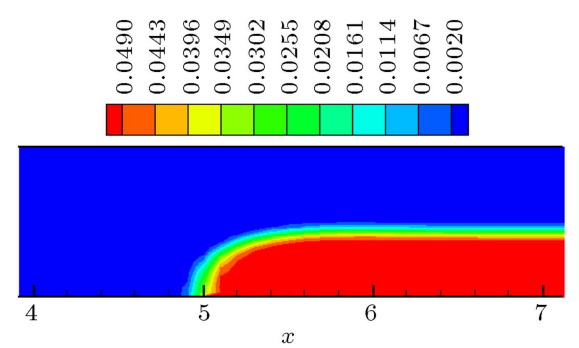

(a)

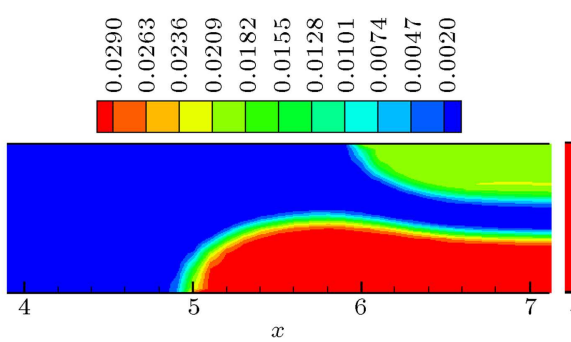

(b)

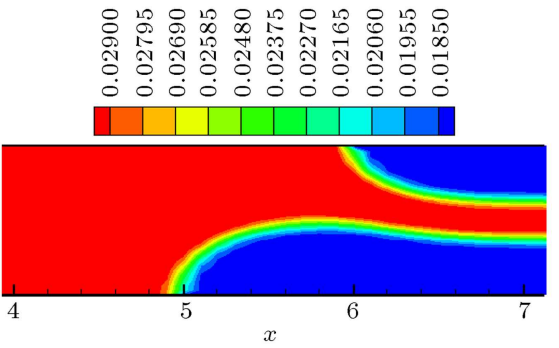

(c)

Figure 8. The contours of nanoparticle volume fraction for double injection: (a) $\phi_{1}=0.0, \phi_{2}=0.0$, and $\phi_{3}=0.05$, (b) $\phi_{1}=0.0, \phi_{2}=0.02$, and $\phi_{3}=0.03$, and (c) $\phi_{1}=0.03, \phi_{2}=0.0182, \phi_{3}=0.0182, \operatorname{Re}_{H_{1}}=0.01$, and $\chi=4$.

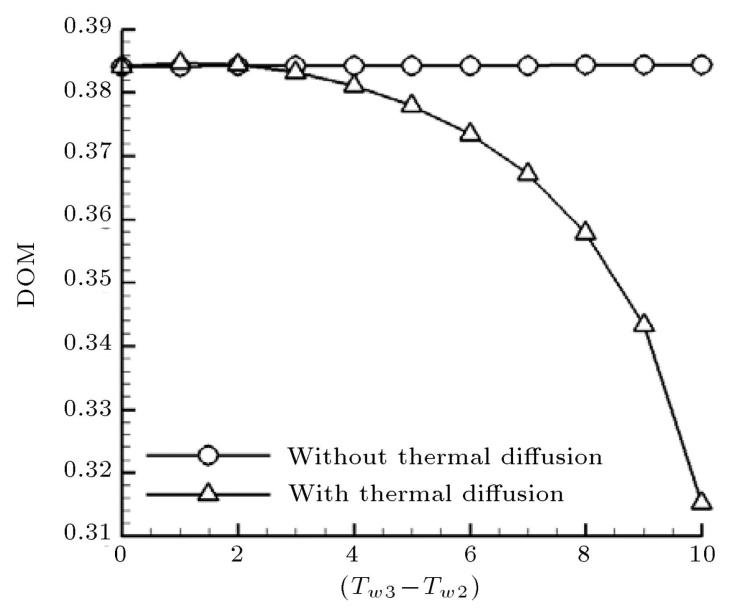

(a)

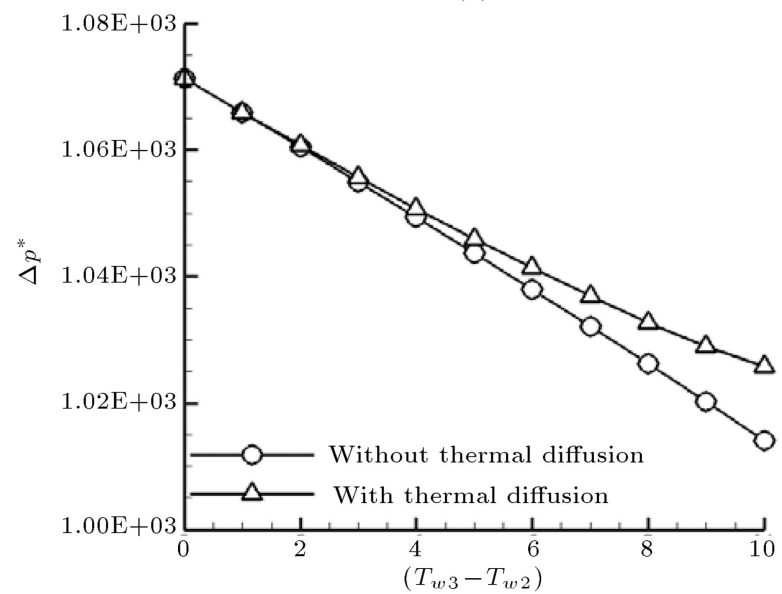

(b)

Figure 9. The effect of temperature dependency of thermophysical properties of nanofluid and thermal diffusion on (a) mixing index, and (b) pressure drop; $l_{2}^{*}=0, \dot{m}_{1}=500 \mu \mathrm{g} / \mathrm{S}, m r=3, \phi_{1}=0, \phi_{2}=0.02$, and $\phi_{3}=0.02$.

since the injection Reynolds numbers are variable with temperature because of temperature dependency of thermophysical properties.

The effect of temperature gradient on mixing index and pressure drop with and without thermal diffusion has been presented in Figure 9. Top and bottom wall temperatures have been named $T_{w 2}$ and

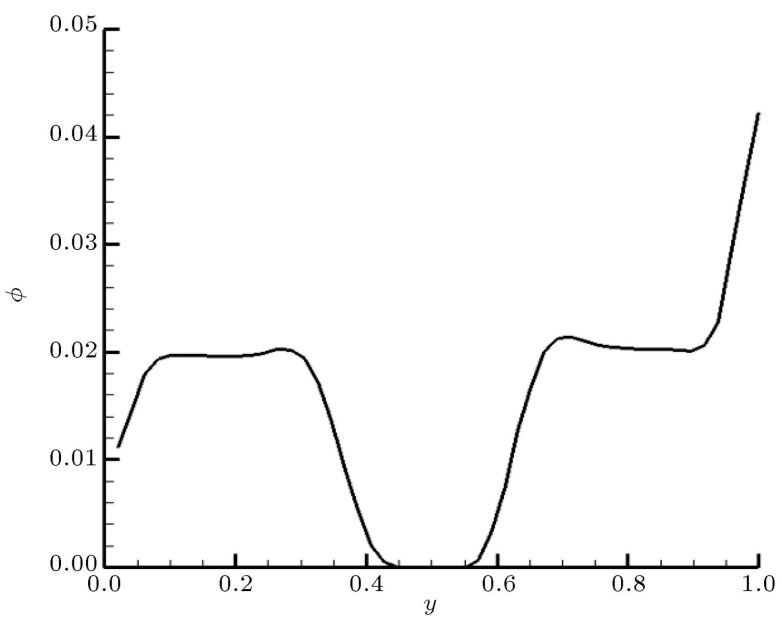

Figure 10. The distribution of nanofluid volume fraction along micromixer exit cross section; $\Delta T=10 \mathrm{~K}, l_{2}^{*}=0$, $\dot{m}_{1}=2500 \mu \mathrm{g} / \mathrm{S}, m r=2, \phi_{1}=0, \phi_{2}=0.02$, and $\phi_{3}=0.02$.

$T_{w 3}$, respectively. Upper wall and injection temperatures have been fixed in $293 \mathrm{~K}$, while bottom wall and injection temperatures are varied. Figure 9 depicts that in absence of thermal diffusion, temperature dependency of thermophysical properties does not have remarkable effect on mixing index; but, in the presence of thermal diffusion, mixing index decreases by increase in temperature difference between upper and lower parts of micromixer. The reason can be obtained from Figure 10, which depicts volume fraction distribution of nanoparticles at exit cross section of micromixer. As can be seen, due to thermal diffusion, nanoparticles have accumulation in some parts of the cross section, which leads to higher variance and less DOM. Pressure drop in all cases decreases by increasing temperature gradient because of reduction in apparent viscosity near the lower wall. The reduction in pressure drop is linear for the case without thermal diffusion, but nonlinear for the case with thermal diffusion.

\subsection{The effect of injection angles}

In all of the above sections, both injection flow angles $\left(\alpha_{2}\right.$ and $\left.\alpha_{3}\right)$ were $90^{\circ}$. In this section, the effect of using various injection angles has been investigated. 


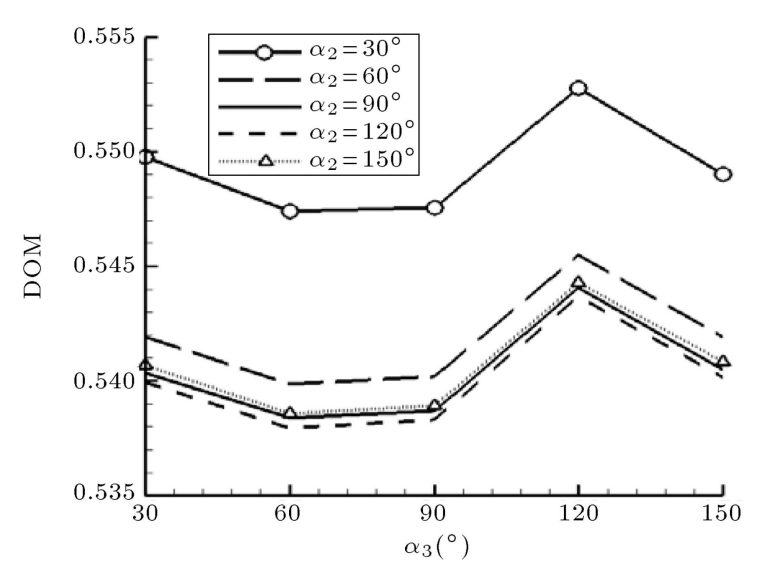

(a)

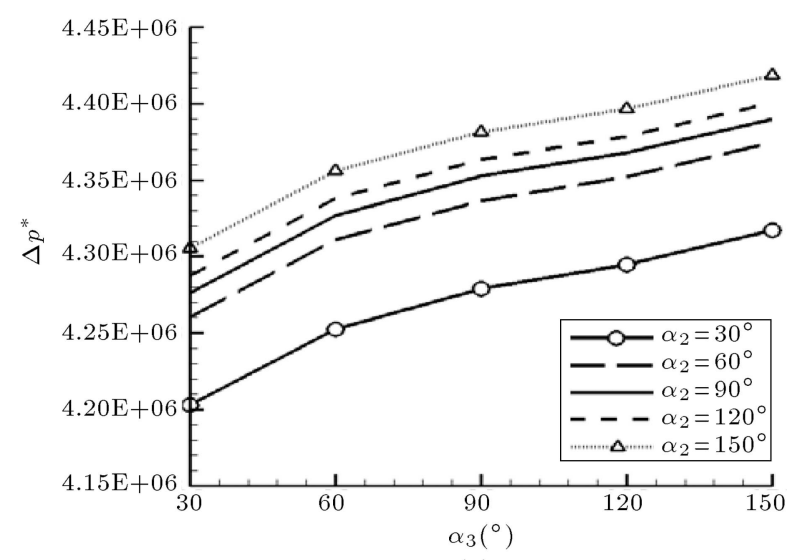

(b)

Figure 11. The effect of injection angles on (a) mixing degree and (b) pressure drop; $l_{2}^{*}=1, \dot{m}_{1}=10 \mu \mathrm{g} / \mathrm{S}$, $m r=3, \phi_{1}=0$, and $\phi_{2}=\phi_{3}=0.02$.

The results have been shown in Figure 11 for degree of mixing and pressure drop. In all cases, mass flow ratio has been maintained at the constant value of $m r=3$.

It can be observed in Figure 11 that for all values of $\alpha_{3}, \alpha_{2}=30^{\circ}$ has the highest degree of mixing. The reason can be obtained from Figure 12. It shows the streamlines for three different angles, colored by volume fraction of nanoparticles. As can be seen, for the special case of $\alpha_{2}=30^{\circ}$ and $\alpha_{3}=120^{\circ}$ (with maximum DOM), mainstream deviates before it reaches the top injection flow. This deviation is due to the pressure reduction near the left-hand-side edge of the top injector. This deviation leads to an increase in convection near the interface between base fluid and nanofluid and, consequently, DOM improvement.

Also, according to Figure 11, the effect of injection angle is not much considerable. In order to show it, since maximum degree of mixing occurs at $\alpha_{3}=120^{\circ}$ for all values of $\alpha_{2}$, streamlines colored by volume fraction of nanoparticles have been presented in Figure 13 for $\alpha_{3}=120^{\circ}$ and all of the studied $\alpha_{2}$ values. It is clear that except for the region near the upper injection fluid, there is not any considerable difference between

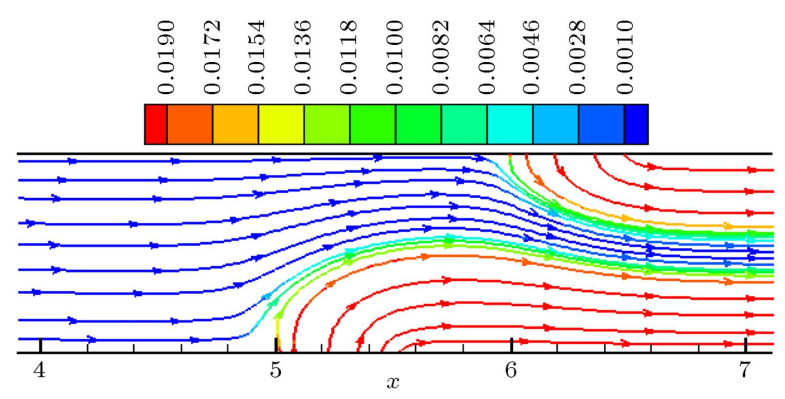

(a)

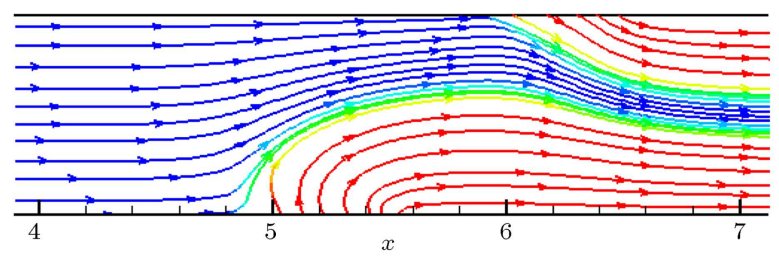

(b)

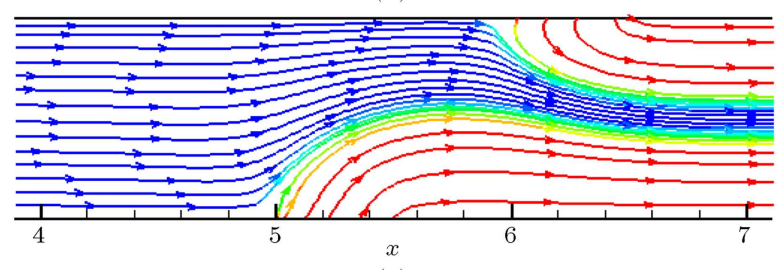

(c)

Figure 12. Streamlines colored by volume fraction of nanoparticles: (a) $\alpha_{2}=\alpha_{3}=90^{\circ}$, (b) $\alpha_{2}=30^{\circ}$, $\alpha_{3}=120^{\circ}$, and (c) $\alpha_{2}=120^{\circ}, \alpha_{3}=60^{\circ}, l_{2}^{*}=1$, $\dot{m}_{1}=10 \mu \mathrm{g} / \mathrm{S}, m r=3, \phi_{1}=0$, and $\phi_{2}=\phi_{3}=0.02$.

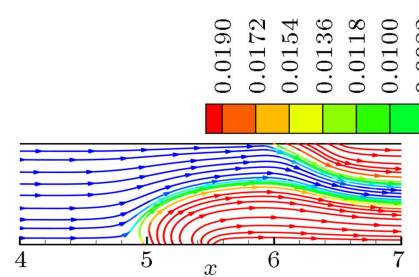

(a)

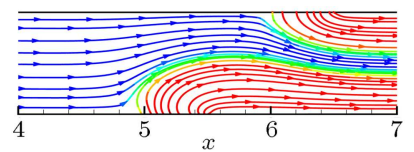

(c)

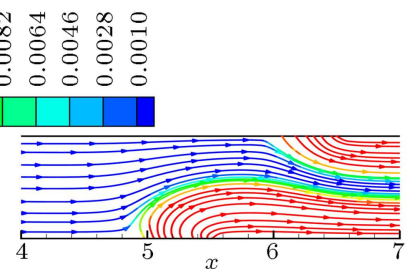

(b)

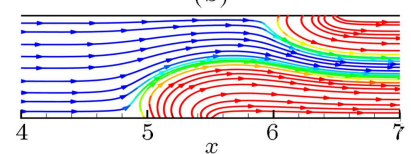

(d)

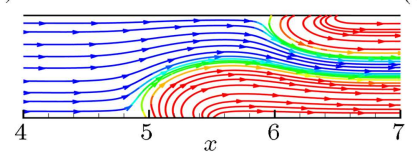

(e)

Figure 13. Streamlines colored by volume fraction of nanoparticles: (a) $\alpha_{2}=30^{\circ}$, (b) $\alpha_{2}=60^{\circ}$, (c) $\alpha_{2}=90^{\circ}$, (d) $\alpha_{2}=120^{\circ}$, and (e) $\alpha_{2}=150^{\circ} ; l_{2}^{*}=1, \dot{m}_{1}=10 \mu \mathrm{g} / \mathrm{S}$, $m r=3, \phi_{1}=0, \phi_{2}=\phi_{3}=0.02$, and $\alpha_{3}=120^{\circ}$.

streamlines and volume fraction contours for various values of $\alpha_{2}$. The small increase in DOM with decrease in $\alpha_{2}$ has been explained above.

Pressure drop varies considerably by variation of injection direction and increases by increase in injection 
angles, because resistance in the mainstream path becomes higher. For $\alpha_{2}=\alpha_{3}=30^{\circ}$, the pressure drop has its minimum value due to the lower shear rate in the interface between base fluid and nanofluid. It can be concluded that injection angle does not have significant effect on mixing index, but has remarkable effect on pressure drop.

\subsection{The effect of baffles}

In this section, effect of height and distance of baffles for double injection in opposite locations has been investigated. Both baffles are located after injections. Effect of baffle height on mixing index and pressure drop has been presented in Figure 14. It can be inferred that mixing index and pressure drop increase by increase in baffle height. Improvement of mixing index is due to increase in the length of diffusion path and expansion of mass boundary layers after baffles. Pressure drop increment is due to higher resistance of nanofluid flow in longer baffle heights.

The effect of distance between baffles on mixing index and pressure drop is illustrated in Figure 15.
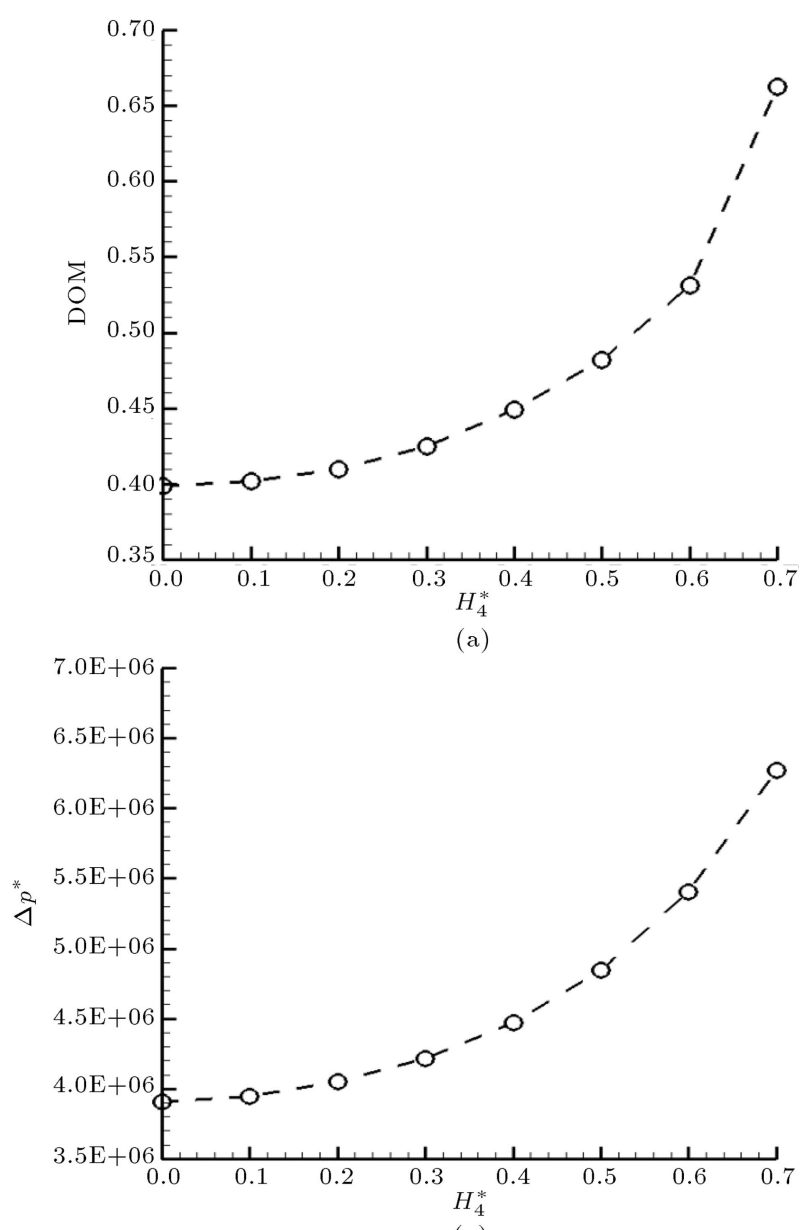

(a)

Figure 14. The effect of height of baffles on (a) mixing index, and (b) pressure drop; $l_{2}^{*}=0, l_{3}^{*}=7, l_{4}^{*}=1$, $m_{1}=10 \mu \mathrm{g} / \mathrm{S}, m r=2, \phi_{1}=0, \phi_{2}=\phi_{3}=0.02$.

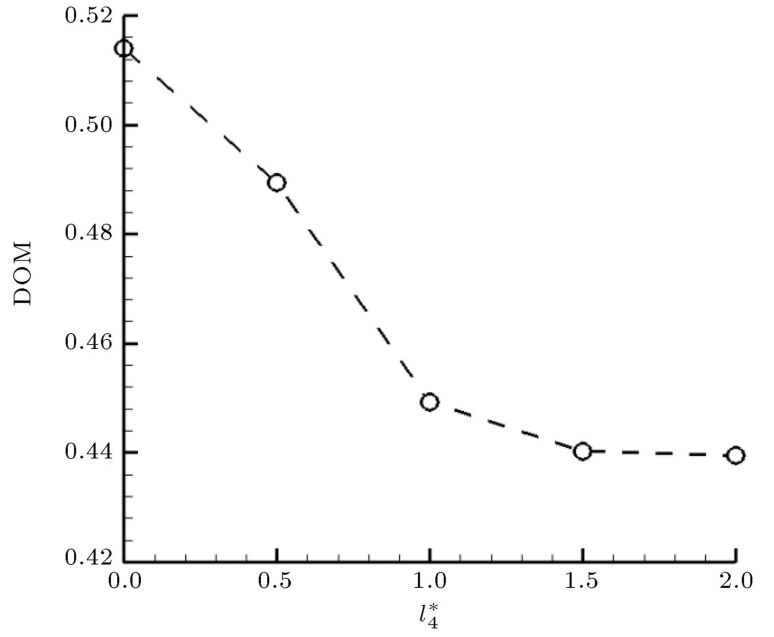

(a)

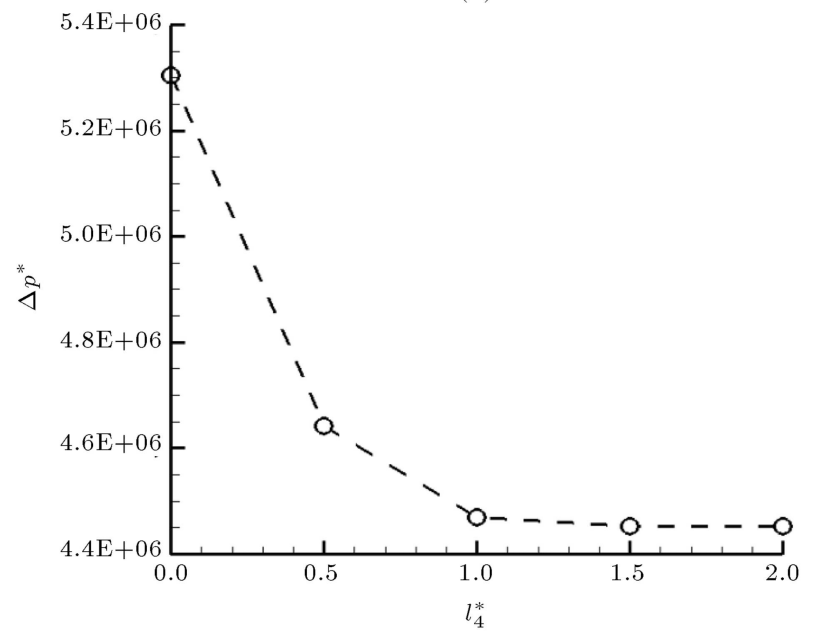

(b)

Figure 15. The effect of distance between baffles on (a) mixing index, and (b) pressure drop; $l_{2}^{*}=0, l_{3}^{*}=7$, $H_{4}^{*}=H_{5}^{*}=0.4, \dot{m}_{1}=10 \mu \mathrm{g} / \mathrm{S}, m r=2, \phi_{1}=0$, $\phi_{2}=\phi_{3}=0.02$.

As we know, contraction-expansion and, therefore, creation of recirculation regions is one of the mixing methods in micromixers. The baffles in this study work according to this mechanism. As can be seen, mixing index decreases by increasing the distance between the baffles. The reason is less expansion of mass boundary layers. Minimum DOM occurs at $l_{4}^{*}=2$ (Figure 15(b)). It seems that $l_{4}^{*}=0.5$ is a turning point (Figure 15(a)).

The behavior can be explained using Figure 16 that shows the velocity magnitude and volume fraction distributions variations versus baffles distance. Figure 16(a) shows that DOM is maximum at $l_{4}^{*}=0$. It is because of the very strong contraction zone between top and bottom baffles. High speed zones at the ends of baffles and, therefore, a recirculating zone behind the baffles were created and they increased the mixing degree. By increasing $l_{4}^{*}=0.5$ from 0 to 0.5 , the magnitude of high speed and strength of recirculating zones decrease and the DOM decreases in comparison 

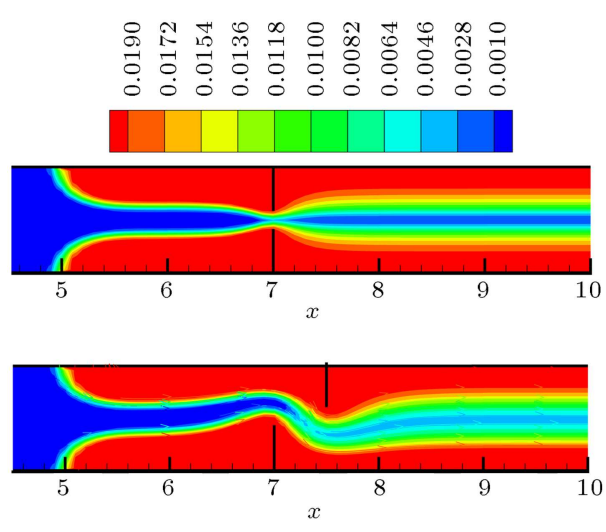

(a)
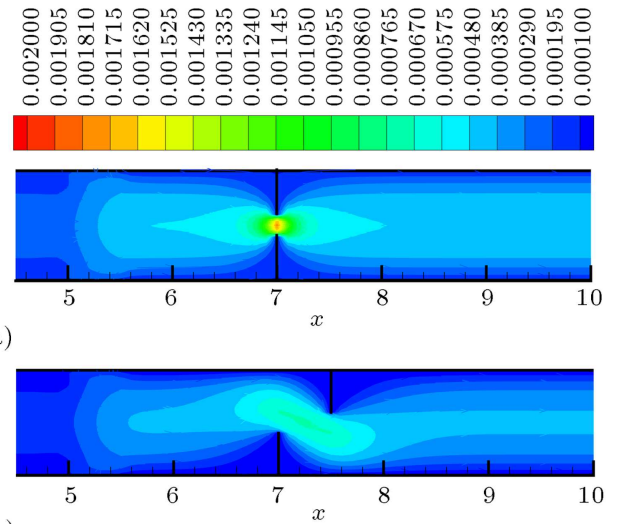

(b)
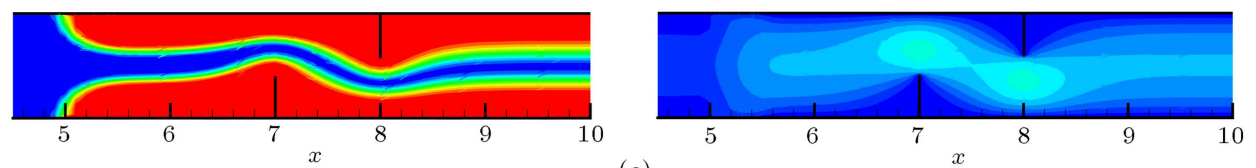

(c)
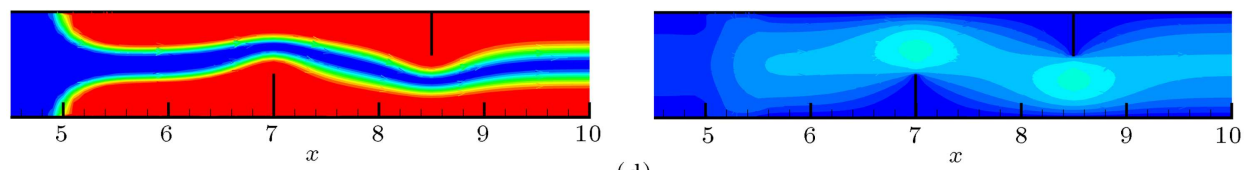

(d)
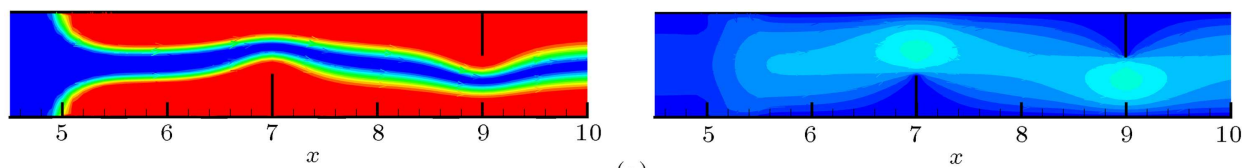

(e)

Figure 16. Distribution of velocity magnitude (right) and volume fraction (left): (a) $l_{4}^{*}=0$, (b) $l_{4}^{*}=0.5,(\mathrm{c}) l_{4}^{*}=1$, (d) $l_{4}^{*}=1.5$, and $(\mathrm{e}) l_{4}^{*}=2, l_{2}^{*}=0, l_{3}^{*}=7, H_{4}^{*}=H_{5}^{*}=0.4, \dot{m}_{1}=10 \mu \mathrm{g} / \mathrm{S}, m r=2, \phi_{1}=0$, and $\phi_{2}=\phi_{3}=0.02$.

to the case with $l_{4}^{*}=0$; but, since the distance between baffles is small, the flow has a sharp turn from the top of bottom baffle toward the bottom of top baffle (Figure 16(b)). As $l_{4}^{*}$ increases, this sharp turning decreases. Therefore, $l_{4}^{*}=0.5$ is the case with maximum variation in velocity and, consequently, maximum variation in mixing degree. This is the reason why $l_{4}^{*}=0.5$ is a turning point.

Pressure drop decreases by increase in the distance between baffles because of less shear rate of nanofluid flow near baffles; however, this reduction is not significant in longer distances.

Finally, baffles have more considerable effect on mixing index than on pressure drop.

\section{Conclusions}

In this study, the laminar mixing of $\mathrm{TiO}_{2} / \mathrm{CMC}$ solution non-Newtonian nanofluid in a passive injection micromixer was numerically studied. The mixture model with variable physical properties of the base fluid was used for simulation of nanofluid flow. A FORTRAN code was written to solve the governing equations by finite volume method. A new method was used for decreasing the computation time. It was observed that double injection produced better mixing and less pressure drop than single injection. Injection orientation has little effect on mixing index, but can affect pressure drop considerably. Effect of temperature on mixing index and pressure drop is important. Volume fraction has considerable effect on mixing index. Use of baffles has significant effect on mixing index and pressure drop and the mixing degree is higher for smaller distances between baffles.

\section{References}

1. Nguyen, N.T. and Wu, Z. "Micromixers - a review", $J$. Micromech. Microeng., 15(2), pp. 1-16 (2005).

2. Engler, M., Kockmann, N., Kiefer, T. and Woias, P. "Numerical and experimental investigations on liquid mixing in static micromixers", Chem. Eng. J., 101(13), pp. $315-322$ (2004).

3. Galletti, M., Roudgar, M., Brunazzi, E. and Mauri, R. "Effect of inlet conditions on the engulfment pattern in a T-shaped micro-mixer", Chem. Eng. J., 185-186, pp. 300-313 (2012).

4. Alam, C. and Kim, K. "Analysis of mixing in a curved 
microchannel with rectangular grooves", Chem. Eng. J., 181-182, pp. 708-716 (2012).

5. Afzal, A. and Kim, K. "Passive split and recombination micromixer with convergent-divergent walls", Chem. Eng. J., 203, pp. 182-192 (2012).

6. Bhagat, A.A.S., Peterson, E. and Papautsky, I. "A passive planar micromixer with obstructions for mixing at low Reynolds numbers", J. Micromech. Microeng., 17(5), pp. 1017-1024 (2007).

7. Ansari, M., Kim, K.Y., Anwar, K. and Kim, S.M. "A novel passive micromixer based on unbalanced splits and collisions of fluid streams", J. Micromech. Microeng., 20(5), p. 055007 (2010).

8. Soleymani, A., Kolehmainen, E. and Turunen, I. "Numerical and experimental investigations of liquid mixing in T-type micromixers", Chem. Eng. J., 135(S1), pp. S219-S228 (2008).

9. Wang, Y., Zhe, J., Chung, B. and Dutta, P. "A rapid magnetic particle driven micromixer", Microfluid. Nanofluid., 4(5), pp. 375-389 (2008).

10. Fu, L.M., Tsai, C.H., Leong, K.P. and Wen, C.Y. "Rapid micromixer via ferrofluids", Phys. Procedia., 9, pp. 270-273 (2010).

11. Bhagat, A.A.S. and Papautsky, I. "Enhancing particle dispersion in a passive planar micromixer using rectangular obstacles", J. Micromech. Microeng., 18(8), p. 085005 (2008).

12. Baheri Islami, S., Dastvareh, B. and Gharraei, R. "An investigation on the hydrodynamic and heat transfer of nanofluid flow with non-Newtonian base fluid in micromixers", Int. J. Heat. Mass Tran., 78, pp. 917929 (2014)

13. Hadigol, M., Nosrati, R., Nourbakhsh, A. and Raisee, M. "Numerical study of electroosmotic micromixing of non-Newtonian fluids", J. Non-Newton. Fluid., 166 (17-18), pp. 965- 971 (2011).

14. Morini, G.L. "Viscous heating in liquid flows in microchannel", Int. J. Heat. Mass Tran., 48(17), pp. 36373647 (2005).

15. Corcione, M., Cianfrini, M. and Quintino, A. "Twophase mixture modeling of natural convection of nanofluids with temperature-dependent properties", Int. J. Therm. Sci., 71, pp. 182-195 (2013).

16. Bird, R.B., Stewert, W.E. and Lightfoot, E.N., Transport Phenomena, 2nd Edn., John Wiley and Sons, USA (2002).

17. Alam, A. and Kim, K.Y. "Mixing performance of a planar micromixer with circular chambers and crossing constriction channels", Sensor. Actuat. B-Chem., 176, pp. 639-652 (2013).

18. Xuan, Y. and Roetzel, W. "Conceptions for heat transfer correlation of Nanofluids", Int. J. Heat. Mass Tran., 43(19), pp. 3701-3707 (2000).

19. Hojjat, M., Etemad, S.Gh., Bagheri, R. and Thibault, J. "Rheological characteristics of non-Newtonian nanofluids: Experimental investigation", Int. J. Heat. Mass Tran., 38(2), pp. 144-148 (2011).

20. Hojjat, M., Etemad, S.Gh., Bagheri, R. and Thibault, J. "Thermal conductivity of non-Newtonian nanofluids: Experimental data and modeling using neural network", Int. J. Heat. Mass Tran., 54(5-6), pp. 10171023 (2011).

21. Einstein, A. "On the movement of small particles suspended in a stationary liquid demanded by the molecular-kinetic theory of heat" [Über die von der molecular kinetischen Theorie der Wärme geforderte Bewegung von in ruhenden Flüssigkeiten suspendierten Teilchen] (in German), Ann. Phys., 17, pp. 549-560 (1905).

22. McNab, G.S. and Meisen, A. "Thermophoresis in liquids", J. Colloid. Interf. Sci., 44(2), pp. 339-346 (1973).

23. Versteeg, H.K. and Malalasekera, W., An Introduction to Computational Fluid Dynamics, 2nd Edn., Pearson, England (2007).

24. Ansys Fluent 12 User's Guide, Fluent Inc. (2009).

\section{Biographies}

Sima Baheri Islami received her BSc degree in 1999 in Mechanical Engineering and MSc degree in 2002 in Thermo-fluid Engineering, both from University of Tabriz, Tabriz, Iran. She is a graduate of University of Tabriz with a PhD in Thermo-fluid Engineering. Currently, she is an Assistant Professor of Thermo-fluid Engineering with Faculty of Mechanical Engineering, University of Tabriz, Iran. Her research interests are non-Newtonian fluids, convective heat transfer, film cooling, microchannels, micromixers, and CFD.

Arash Alizad received his BSc and MSc degrees both in Mechanical Engineering from University of Tabriz, Tabriz, Iran, in 2012 and 2014, respectively. His research interests include computational fluid dynamics, non-Newtonian fluids, nanofluids, and deformable particles in different types of flows.

Reza Gharraei received his BSc degree in Mechanical Engineering from University of Tabriz, Tabriz, Iran, in 2001; MSc degree in Thermo-fluid Engineering from Amirkabir University of Technology, Tehran, Iran, in 2004; and PhD degree in Thermo-fluid Engineering from University of Tabriz, Tabriz, Iran, in 2010. Currently, he is an Assistant Professor with Mechanical Engineering Department, Azarbaijan Shahid Madani University, Tabriz, Iran. His research interests are twophase flow, electrohydrodynamics, heat transfer and fluid flow in microchannels, and non-Newtonian fluids flow. 